\title{
Total synthesis of elansolids B1 and B2
}

\author{
Liang-Liang Wang and Andreas Kirschning*
}

\section{Full Research Paper}

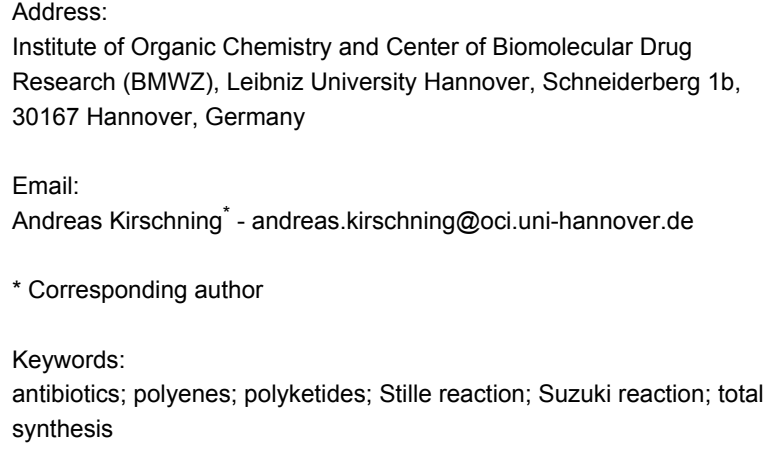

Beilstein J. Org. Chem. 2017, 13, 1280-1287.

doi:10.3762/bjoc. 13.124

Received: 03 April 2017

Accepted: 17 May 2017

Published: 28 June 2017

This article is part of the Thematic Series "Lipids: fatty acids and derivatives, polyketides and isoprenoids".

Guest Editor: J. S. Dickschat

(C) 2017 Wang and Kirschning; licensee Beilstein-Institut. License and terms: see end of document.

\begin{abstract}
The elansolids A1-A3, B1, and B2 are secondary metabolites formed by the gliding bacterium Chitinophaga sancti. They show antibacterial activity against Gram-positive bacteria. A second generation total synthesis of the antibiotic elansolid B1 (2) and the first synthesis of elansolid B2 (3) are reported. In contrast to previous work, the (Z,E,Z)-triene at C10-C15 was assembled by using an optimized $\mathrm{C}-\mathrm{C}$ cross-coupling sequence with a Suzuki cross-coupling reaction as key step.
\end{abstract}

\section{Introduction}

The elansolids are metabolites from the gliding bacterium Chitinophaga sancti (formerly Flexibacter spec.) (Figure 1) $[1,2]$. Elansolid A2 $\left(\mathbf{1}^{*}\right)$, an atropisomer of elansolid A1 (1), showed antibiotic activity against Gram-positive bacteria in the range of 0.2 to $64 \mu \mathrm{g} / \mathrm{mL}$ and cytotoxicity against $\mathrm{L} 929$ mouse fibroblast cells with an $\mathrm{IC}_{50}$ value of $12 \mu \mathrm{g} / \mathrm{mL}$. Besides these two macrocylic members also elansolids B1 (2) and B2 (3) along with A3 (4) bearing the unusual $p$-quinone methide unit were isolated from the fermentation broth. All elansolids belong to the group of trans-polyketides type I [3-6].

For the first generation total synthesis of elansolid B1 (2) we utilized an endo-selective intramolecular Diels-Alder (IMDA) cycloaddition as key step to construct the tetrahydroindane unit (Scheme 1) [7]. An enone, derived from allylic alcohol 8 served as precursor to yield tetrahydroindane $\mathbf{9}$ with excellent diastereocontrol at $-25{ }^{\circ} \mathrm{C}$. The major drawback of our first total synthesis of elansolid B1 (2) was the installation of the side chain at $\mathrm{C} 1-\mathrm{C} 13$. The synthesis relied on two consecutive Sonogashira-Hagihara cross-coupling reactions that provided the ene-diyne system (C10-C15) 10 in good yield. However, partial hydrogenation (only the zinc-copper couple worked) furnished the desired (Z,E,Z)-triene 11 in only low yield (35\%) and overreduction was difficult to control. Practically, the reduction was stopped when still substantial amounts of monoreduced product (the alkyne at $\mathrm{C} 10-\mathrm{C} 11$ is reduced pref- 

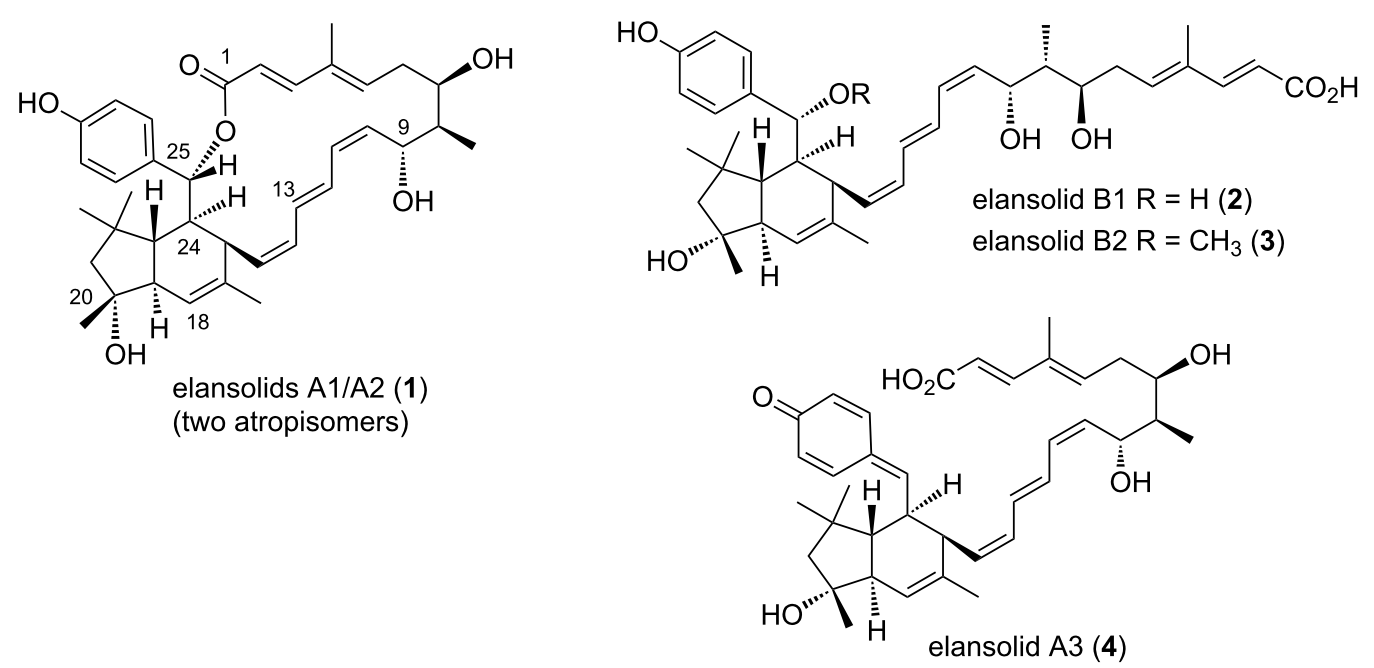

Figure 1: Elansolids A1/A2, B1, B2 and A3 (1-4).
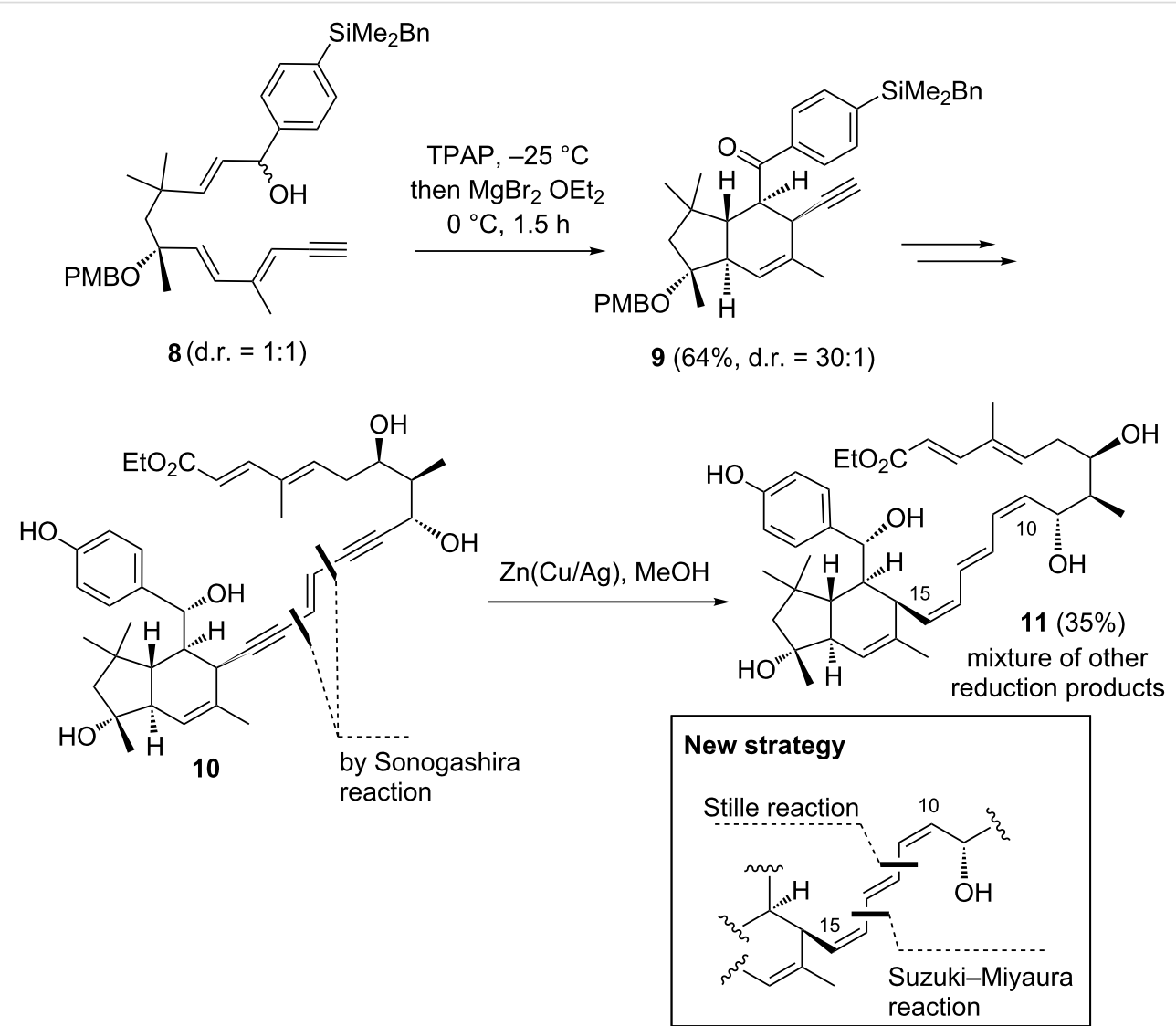

Scheme 1: IMDA to generate the tetrahydroindane unit of the elansolids by oxidation of benzyl ether 8 as precursor and construction of the (Z,E,Z)triene unit at $\mathrm{C} 10-\mathrm{C} 15$ (TPAP = tetra- $n$-propylammonium perruthenate(VII); PMB = p-methoxybenzyl; $\mathrm{Bn}=$ benzyl).

erentially) were present. Consequently, the hydrogenation yielded a mixture of products, which in any case made the separation and isolation a very challenging task.
As continuation of our synthetic investigations on the elansolids, we report an improved second generation approach for generating the carbon chain $\mathrm{C} 1-\mathrm{C} 13$ [8] and for preparing elan- 
solid B1 (2). Furthermore, we also describe the first synthesis of elansolid B2 (3). The key for improvement was to abandon the two Sonogashira reactions along with the syn-reductions of the two alkynes. Instead, we planned to utilize the Suzuki-Miyaura and the Stille reactions and two $Z$-configured vinyl iodides to assemble the $(Z, E, Z)$-triene unit.

\section{Results and Discussion}

The improved synthesis utilizes the Suzuki-Miyaura cross-coupling reaction to merge the western fragment derived from ketone 9 with the newly designed eastern building block 13. This fragment was obtained in very good yield from vinyl iodide $\mathbf{1 2}$ [9] by a Stille protocol using doubly functionalized alkene $\mathbf{1 4}$ which is suited for a sequential cross-coupling strategy (Scheme 2). Under the catalytic conditions, we did not encounter isomerization of the alkene and diene configurations in vinyl boronate 13 .

The preparation of the newly modified western fragment started from known IMDA product 9 [7], which was first reduced at C-25 (Scheme 3). The two diastereoisomers could be separated by chromatography and the stereochemical assignment of the major isomer was based on X-ray crystallographic analysis [7]. Next, Tamao-Fleming oxidation [10] yielded phenol 15. The alkyne was transformed into vinyl iodide $\mathbf{1 7}$ after $O$-acylation, iodination of the terminal alkyne and finally diimide-mediated syn-reduction [11].

Next, DDQ-mediated removal of the PMB protecting group yielded vinyl iodide 18. The synthesis of both fragments $\mathbf{1 3}$ and 18 set the stage for the Suzuki-Miyaura coupling which delivered the desired $(Z, E, Z)$-configured triene 19. Again, we did not encounter formation of stereoisomers in the triene unit. The configuration of the triene was unequivocally assigned by analysis of coupling constants $(J)$ and by measuring nuclear Overhauser effects (nOe). Finally, desilylation and global saponification of all ester groups in the presence of isopropanol success- fully yielded elansolid B1 (2). When isopropanol was exchanged by methanol, elansolid B2 (3) was generated. Its formation can be rationalized by formation of the intermediate $p$-methide quinone which selectively trapped methanol, exclusively yielding the $R$-isomer at $\mathrm{C} 25$. This excellent facial selectivity has been demonstrated, e.g., for anilines as nucleophiles before. It is due to the preferred conformation around the bond at $\mathrm{C} 24-\mathrm{C} 25$ which leads to the efficient shielding of the si-face by the two germinal methyl groups at C22 [4,5]. The NMR data determined for both synthetic products were identical with those of authentic samples of elansolid B1 (2) and elansolid B2 (3) (copies of spectra, see Supporting Information File 1).

\section{Conclusion}

In conclusion, we describe an improved second generation synthesis of the highly active antibiotic elansolid B1 (2). The improvements are mainly associated with the preparation of the triene unit at $\mathrm{C} 10-\mathrm{C} 15$ by utilizing the Stille and the Suzuki-Miyaura cross-coupling reactions as well as the highly versatile difunctionalized building block 14. In principal, the synthesis sheds light on how such $(Z, E, Z)$-configured triene units are ideally be constructed, clearly demonstrating that enediynes are less preferred precursors for such structural elements. It has to be noted that there is precedence in the literature for the use of the Suzuki-Miyaura cross-coupling reaction as key step to assemble differently configured trienes present in polyketides [12-15].

Furthermore, we show how the intermediate $p$-methide quinone can be exploited to also prepare elansolid B2 (3). The improved synthesis allows more easily preparing analogues of the elansolids for further biological evaluation.

\section{Experimental General information:}

${ }^{1} \mathrm{H}$ NMR spectra were recorded at $400 \mathrm{MHz}$ or $500 \mathrm{MHz}$, respectively, and ${ }^{13} \mathrm{C}$ NMR spectra were recorded at $100 \mathrm{MHz}$ or<smiles>CCOC(=O)/C=C/C(C)=C/C[C@@H](OCC)[C@@H](C)[C@H](OCC)[C@H](/C=C\C=C\B1OC(C)(C)C(C)(C)O1)OCC</smiles> 


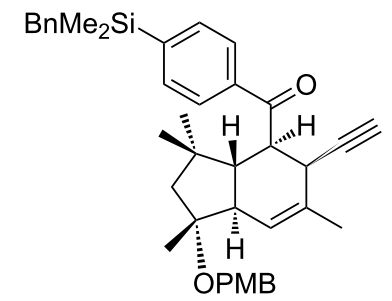

9
1. $\mathrm{LiAlH}_{4}, \mathrm{THF},-70^{\circ} \mathrm{C}$ $(80 \%$, d.r. $=3: 1)$

2. TBAF, $\mathrm{H}_{2} \mathrm{O}_{2}$, $\mathrm{THF} / \mathrm{MeOH}$, rt (99\%)

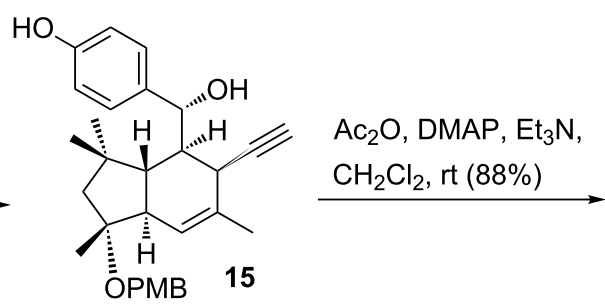

$\mathrm{AcO}$

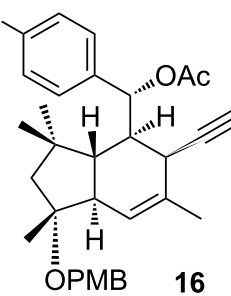

1. $\mathrm{AgNO}_{3}, \mathrm{NIS}$, acetone, $\mathrm{rt}(84 \%)$

$\mathrm{AcO}$

\section{2. $\mathrm{NBSH}, \mathrm{Et}_{3} \mathrm{~N}, \mathrm{iPrOH} / \mathrm{THF}$, rt $(77 \%)$}

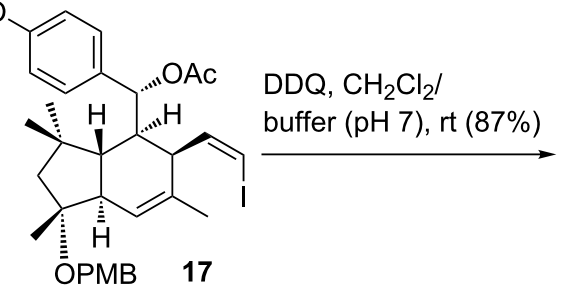

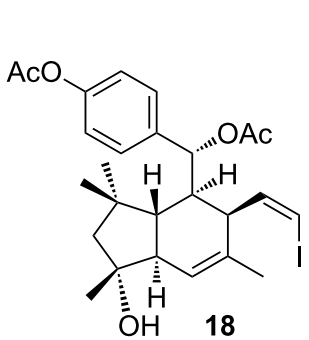

$\mathrm{Pd}\left(\mathrm{PPh}_{3}\right)_{4}, \mathrm{Tl}_{2} \mathrm{CO}_{3}$, $\mathrm{THF} / \mathrm{H}_{2} \mathrm{O}$, rt $(77 \%)$
$\mathrm{AcO}$<smiles>[C]O[Co]</smiles>

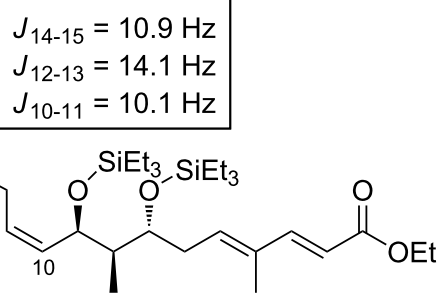

$J_{14-15}=10.9 \mathrm{~Hz}$

$J_{12-13}=14.1 \mathrm{~Hz}$<smiles>C=C1C[C@H](C)[IH][C@H](CC)[C@H](OC(=C)C)C1</smiles>

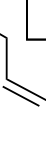

19

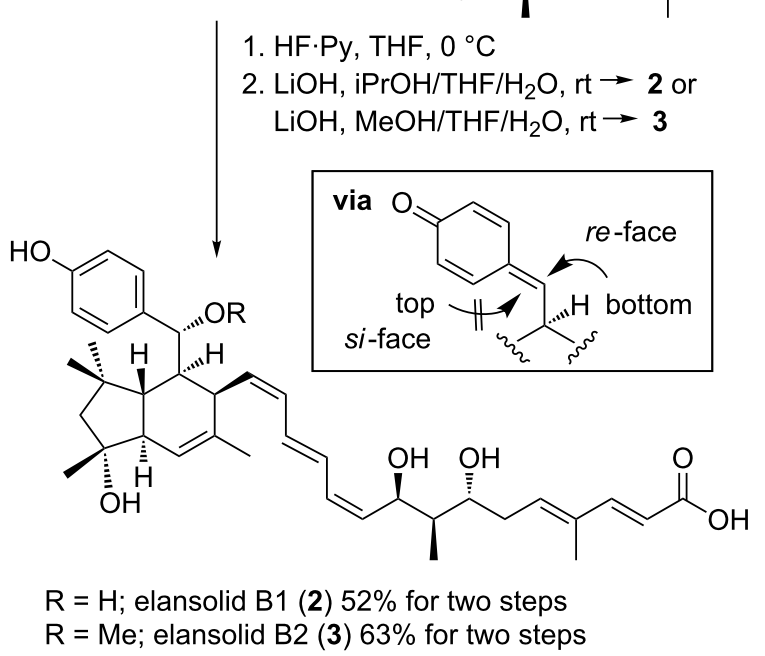

1. HF.Py, THF, $0^{\circ} \mathrm{C}$

$\mathrm{LiOH}, \mathrm{iPrOH} / \mathrm{THF} / \mathrm{H}_{2} \mathrm{O}$, rt $\rightarrow 2$ or<smiles>CCO[B]/C=C\C=C/[C@H](OCC)[C@@H](C)[C@H](C/C=C(C)/C=C/C(=O)OCC)OCC</smiles>
13

Scheme 3: Total synthesis of elansolids B1 (2) and B2 (3).

$125 \mathrm{MHz}$, respectively, with a Bruker Avance 400, DPX 400 or DRX 500. Chemical shift values of NMR data are reported as values in ppm relative to the (residual undeuterated) solvent signal as internal standard. Multiplicities for ${ }^{1} \mathrm{H}$ NMR signals are described using the following abbreviations: $\mathrm{s}=$ singlet, $\mathrm{d}=$ doublet, $\mathrm{t}=$ triplet, $\mathrm{q}=$ quartet, $\mathrm{m}=$ multiplet; where appropriate with the addition of $b=$ broad. Mass spectra were obtained with a type LCT (ESI) (Micromass) equipped with a lockspray dual ion source in combination with a Waters
Alliance 2695 LC system, or with a type QTOF premier (Micromass) spectrometer (ESI mode) in combination with a Waters Acquity UPLC system equipped with a Waters BEH C18 $1.7 \mu \mathrm{m}$ (SN 01473711315545) column (solvent A: water $+0.1 \%(\mathrm{v} / \mathrm{v})$ formic acid, solvent $\mathrm{B}: \mathrm{MeOH}+0.1 \%(\mathrm{v} / \mathrm{v})$ formic acid; flow rate $=0.4 \mathrm{~mL} / \mathrm{min}$; gradient $(t[\mathrm{~min}] /$ solvent $\mathrm{B}$ [\%]): (0:5) (2.5:95) (6.5:95) (6.6:5) (8:5)). Ion mass signals $(\mathrm{m} / \mathrm{z})$ are reported as values in atomic mass units. Optical rotations were measured on a Perkin-Elmer polarimeter type 341 or 
241 in a quartz glass cuvette at $l=589 \mathrm{~nm}(\mathrm{Na} \mathrm{D}$-line). The optical rotation is given in $\left[{ }^{\circ} \mathrm{mL} \cdot \mathrm{g}^{-1} \cdot \mathrm{dm}^{-1}\right]$ with $c=1$ corresponding to $10 \mathrm{mg} \mathrm{mL}^{-1}$. Preparative HPLC was operated at a Merck Hitachi LaChrome HPLC (Pump L7150 or L7100, Interface D-7000, Diode Array Detector L-7450), respectively, at a Beckmann system Gold HPLC (Solvent Module 125, Detector 166). Solvents, columns, operating procedures and retention times are given with the corresponding experimental and analytical data.

All reactions were performed under an argon atmosphere unless otherwise stated. Glassware was dried by heating under vacuum followed by flushing with argon gas prior to use. Dry solvents were obtained after filtration through drying columns on a M. Braun solvent purification system or purchased form commercial providers. The synthesis of building blocks 9 [7] and 12 [9] was reported before.

\section{Synthetic experiments \\ Synthesis of boronate 13}

A flame dried round bottom flask equipped with a stirring bar was charged with vinyl iodide 12 ( $10 \mathrm{mg}, 16 \mu \mathrm{mol}, 1.0$ equiv) and boronate 14 (10.68 mg, $24 \mu \mathrm{mol}, 1.5$ equiv) in DMF $(0.3 \mathrm{~mL})$ and THF $(0.1 \mathrm{~mL})$. To this stirred solution $\mathrm{PdCl}_{2}\left(\mathrm{MeCN}_{2}(1.25 \mathrm{mg}, 4.8 \mu \mathrm{mol}, 0.3\right.$ equiv) was added at room temperature and stirring was continued for $8 \mathrm{~h}$. The volatiles were removed in vacuo and the residue was purified by silica gel chromatography to afford vinyl boronate $\mathbf{1 3}(8.5 \mathrm{mg}$, $12.3 \mu \mathrm{mol}, 82 \%) . R_{\mathrm{f}}=0.40$ (PE/EtOAc 10:1, visualized using an anisaldehyde stain or UV), $[\alpha]_{\mathrm{D}}{ }^{20}=-34.5\left(c 0.8, \mathrm{CH}_{2} \mathrm{Cl}_{2}\right)$; ${ }^{1} \mathrm{H} \mathrm{NMR}\left(400 \mathrm{MHz}, \mathrm{CDCl}_{3}, \mathrm{CHCl}_{3}=7.26 \mathrm{ppm}\right) \delta 7.32(\mathrm{~d}, J=$ $15.6 \mathrm{~Hz}, 1 \mathrm{H}), 7.25(\mathrm{~d}, J=11.4,17.5 \mathrm{~Hz}, 1 \mathrm{H}), 6.09(\mathrm{t}, J=11.1$ $\mathrm{Hz}, 1 \mathrm{H}), 5.97$ (dd, $J=7.1 \mathrm{~Hz}, 1 \mathrm{H}), 5.78(\mathrm{~d}, J=15.5 \mathrm{~Hz}, 1 \mathrm{H})$, $5.61(\mathrm{~d}, J=17.4 \mathrm{~Hz}, 1 \mathrm{H}), 5.57(\mathrm{t}, J=10.2 \mathrm{~Hz}, 1 \mathrm{H}), 4.63(\mathrm{dd}, J$ $=6.4,9.2 \mathrm{~Hz}, 1 \mathrm{H}), 4.22(\mathrm{q}, J=7.1 \mathrm{~Hz}, 2 \mathrm{H}), 3.84-3.80(\mathrm{~m}, 1 \mathrm{H})$, 2.42-2.34 (m, 1H), 2.27-2.23 (m, 1H), $1.76(\mathrm{~s}, 3 \mathrm{H}), 1.74-1.71$ $(\mathrm{m}, 1 \mathrm{H}), 1.33(\mathrm{t}, J=7.2 \mathrm{~Hz}, 3 \mathrm{H}), 1.28(\mathrm{~s}, 12 \mathrm{H}), 0.97-0.93(\mathrm{~m}$, $21 \mathrm{H}), 0.59(\mathrm{q}, J=8.0 \mathrm{~Hz}, 12 \mathrm{H}) ;{ }^{13} \mathrm{C} \mathrm{NMR}(100 \mathrm{MHz}$, $\left.\mathrm{CDCl}_{3}=77.16 \mathrm{ppm}\right) \delta 167.6,149.6,143.8,139.6,137.4,133.6$, 130.6, 123.3, 115.4, 83.2, 72.5, 69.8, 60.1, 47.1, 32.6, 24.8, $24.7,14.3,12.6,9.7,7.0,6.9,5.2,5.1$; HRMS (ESI) $m / z:[\mathrm{M}+\mathrm{Na}]^{+}$calculated for $\mathrm{C}_{35} \mathrm{H}_{65} \mathrm{BO}_{6} \mathrm{Si}_{2} \mathrm{Na}, 671.4310$; found, 671.4312 .

\section{Reduction of ketone 9 and formation of benzyl alcohol}

A solution of ketone 9 (466.2 $\mathrm{mg}, 0.79 \mathrm{mmol}, 1.0$ equiv) in THF (3 mL) was added to a suspension of $\mathrm{LiAlH}_{4}(599.2 \mathrm{mg}$, $15.79 \mathrm{mmol}, 20$ equiv) in THF $(12 \mathrm{~mL})$ at $-70{ }^{\circ} \mathrm{C}$. After stirring for $3 \mathrm{~d}$ at this temperature the reaction was terminated by slow addition of a saturated potassium sodium tartrate solution.
The reaction mixture was vigorously stirred for $1 \mathrm{~h}$ at room temperature. The layers were seperated and the aqueous phase was extracted with ethyl acetate. The combined organic extracts were dried over $\mathrm{Na}_{2} \mathrm{SO}_{4}$, filtered and concentrated under reduced pressure. The resulting benzyl alcohol $(371.7 \mathrm{mg}$, $0.63 \mathrm{mmol}, 80 \%$, d.r. $=3: 1$ ) was obtained after flash column chromatography. Separation of diastereomers was achieved by preparative HPLC (C18 ISIS-SP) (gradient $\mathrm{H}_{2} \mathrm{O} / \mathrm{MeOH}$ 30:70 to $\left.0: 100\{0-80 \mathrm{~min}\}, 15 \mathrm{~mL} / \mathrm{min}, t_{\mathrm{R}}=67.6 \mathrm{~min}\right) . R_{\mathrm{f}}=0.6(\mathrm{PE} /$ EtOAc 5:1, visualized using anisaldehyde stain or $\mathrm{UV})$, $[\alpha]_{\mathrm{D}}{ }^{20}=+28.9\left(c 0.82, \mathrm{CH}_{2} \mathrm{Cl}_{2}\right) ;{ }^{1} \mathrm{H} \mathrm{NMR}\left(400 \mathrm{MHz}, \mathrm{CDCl}_{3}\right.$, $\left.\mathrm{CHCl}_{3}=7.26 \mathrm{ppm}\right) \delta 7.48(\mathrm{~d}, J=8.0 \mathrm{~Hz}, 2 \mathrm{H}), 7.42(\mathrm{~d}, J=7.9$ $\mathrm{Hz}, 2 \mathrm{H}), 7.30$ (d, $J=9.8 \mathrm{~Hz}, 2 \mathrm{H}), 7.19(\mathrm{t}, J=7.5 \mathrm{~Hz}, 2 \mathrm{H}), 7.08$ $(\mathrm{t}, J=7.3 \mathrm{~Hz}, 1 \mathrm{H}), 6.94-6.89(\mathrm{~m}, J=6.8 \mathrm{~Hz}, 4 \mathrm{H}), 5.60(\mathrm{~s}, 1 \mathrm{H})$, $5.29(\mathrm{dd}, J=2.9,11.0 \mathrm{~Hz}, 1 \mathrm{H}), 4.47(\mathrm{~d}, J=10.8 \mathrm{~Hz}, 1 \mathrm{H}), 4.40$ $(\mathrm{d}, J=10.8 \mathrm{~Hz}, 1 \mathrm{H}), 4.27(\mathrm{~d}, J=11.1 \mathrm{~Hz}, 1 \mathrm{H}, \mathrm{OH}), 3.83$ (s, $3 \mathrm{H}), 2.87$ (d, $J=10.7 \mathrm{~Hz}, 1 \mathrm{H}), 2.57$ (br, 1H), 2.48 (d, $J=2.4$ $\mathrm{Hz}, 1 \mathrm{H}), 2.33(\mathrm{~s}, 2 \mathrm{H}), 2.20(\mathrm{ddd}, J=3.5,4.6,11.4 \mathrm{~Hz}, 1 \mathrm{H})$, $2.10(\mathrm{~d}, J=14.0 \mathrm{~Hz}, 1 \mathrm{H}), 2.02(\mathrm{dd}, J=11.8,12.0 \mathrm{~Hz}, 1 \mathrm{H})$, $1.69(\mathrm{~s}, 3 \mathrm{H}), 1.69(\mathrm{~d}, J=13.6 \mathrm{~Hz}, 1 \mathrm{H}), 1.40(\mathrm{~s}, 3 \mathrm{H}), 1.32$ (s, $3 \mathrm{H}), 1.29(\mathrm{~s}, 3 \mathrm{H}), 0.29(\mathrm{~s}, 6 \mathrm{H}) ;{ }^{13} \mathrm{C} \mathrm{NMR}(100 \mathrm{MHz}$, $\left.\mathrm{CDCl}_{3}=77.16 \mathrm{ppm}\right) \delta 158.9,144.3,139.7,136.6,133.8,133.2$, $131.8,128.7,128.3,128.1,124.7,124.1,123.7,113.8,86.7$, $81.3,73.9,73.5,64.9,55.8,55.3,52.9,46.6,45.3,38.0,33.4$, $31.1,26.3,24.2,22.7,21.3,-3.4,-3.3$; HRMS (ESI) $m / z:[\mathrm{M}+\mathrm{Na}]^{+}$calculated for $\mathrm{C}_{39} \mathrm{H}_{48} \mathrm{O}_{3} \mathrm{SiNa}, 615.3270$; found, 615.3270 .

\section{Synthesis of phenol 15}

To a solution of the benzyl alcohol described above $(124.0 \mathrm{mg}$, $0.21 \mathrm{mmol}, 1.0$ equiv) in THF $(0.75 \mathrm{~mL})$ was added a solution of TBAF ( $c=1.0 \mathrm{M}$ in THF, $0.84 \mathrm{~mL}, 4.0$ equiv) and the mixture was stirred for $15 \mathrm{~min}$. Methanol $(2.23 \mathrm{~mL}), \mathrm{KHCO}_{3}$ (41.85 mg, 0.42 mmol, 2.0 equiv) and $\mathrm{H}_{2} \mathrm{O}_{2}\left(35 \%\right.$ in $\mathrm{H}_{2} \mathrm{O}$, $0.36 \mathrm{~mL}, 4.18 \mathrm{mmol}, 20$ equiv) were sequentially added and stirring was continued overnight. Then, the reaction was terminated by slow addition of a saturated, aqueous $\mathrm{Na}_{2} \mathrm{~S}_{2} \mathrm{O}_{3}$ solution and the aqueous layer was extracted with ethyl acetate. The combined organic extracts were dried over $\mathrm{Na}_{2} \mathrm{SO}_{4}$ filtered and concentrated in vacuo. Purification by flash column chromatography gave phenol $15(96.0 \mathrm{mg}, 0.20 \mathrm{~mol}, 99 \%)$ as a colorless solid. $R_{\mathrm{f}}=0.20$ (PE/EtOAc 3:1, visualized using an anisaldehyde stain or UV), $[\alpha]_{\mathrm{D}}{ }^{20}=+26.1\left(c 0.7, \mathrm{CH}_{2} \mathrm{Cl}_{2}\right) ;{ }^{1} \mathrm{H} \mathrm{NMR}$ (400 MHz, $\left.\mathrm{CDCl}_{3}, \mathrm{CHCl}_{3}=7.26 \mathrm{ppm}\right) \delta 7.31-7.28(\mathrm{~m}, 4 \mathrm{H})$, $6.90(\mathrm{~d}, J=8.7 \mathrm{~Hz}, 2 \mathrm{H}), 6.86(\mathrm{~d}, J=8.6 \mathrm{~Hz}, 2 \mathrm{H}), 5.59(\mathrm{~s}, 1 \mathrm{H})$, $5.25(\mathrm{dd}, J=3.0,10.9 \mathrm{~Hz}, 1 \mathrm{H}), 4.97(\mathrm{~s}, 1 \mathrm{H}, \mathrm{OH}), 4.47$ (d, $J=$ $10.8 \mathrm{~Hz}, 1 \mathrm{H}), 4.39$ (d, $J=10.8 \mathrm{~Hz}, 1 \mathrm{H}), 4.22(\mathrm{~d}, J=10.9 \mathrm{~Hz}$, $1 \mathrm{H}), 3.82$ (s, 3H), 2.85 (dd, $J=1.8,12.4 \mathrm{~Hz}, 1 \mathrm{H}), 2.60$ (br, 1H), $2.45(\mathrm{~d}, J=2.5 \mathrm{~Hz}, 1 \mathrm{H}), 2.13(\mathrm{ddd}, J=3.8,4.4,11.1 \mathrm{~Hz}, 1 \mathrm{H})$, $2.08(\mathrm{~d}, J=14.0 \mathrm{~Hz}, 1 \mathrm{H}), 2.00(\mathrm{t}, J=11.8 \mathrm{~Hz}, 1 \mathrm{H}), 1.68$ (s, $3 \mathrm{H}), 1.67(\mathrm{~d}, J=13.7 \mathrm{~Hz}, 1 \mathrm{H}), 1.38(\mathrm{~s}, 3 \mathrm{H}), 1.30$ (s, 3H), 1.28 
(s, 3H); ${ }^{13} \mathrm{C} \mathrm{NMR}\left(100 \mathrm{MHz}, \mathrm{CDCl}_{3}=77.16 \mathrm{ppm}\right) \delta 158.9$, $154.4,135.5,133.2,131.8,128.7,126.6,123.7,115.2,113.8$, 86.8, 81.3, 73.9, 73.1, 64.9, 55.7, 55.3, 52.9, 46.7, 45.4, 38.0, $33.3,31.1,24.2,22.7,21.3$; HRMS (ESI) $m / z:[\mathrm{M}+\mathrm{H}]^{+}$calculated for $\mathrm{C}_{30} \mathrm{H}_{37} \mathrm{O}_{4}, 461.2692$; found, 461.2693 .

\section{Synthesis of acetyl ester 16}

A round bottom flask equipped with a magnetic stirring bar was sequentially charged with alcohol $15(89.6 \mathrm{mg}, 0.195 \mathrm{mmol}$, 1.0 equiv), $\mathrm{CH}_{2} \mathrm{Cl}_{2}(2 \mathrm{~mL})$, DMAP (23.82 mg, $0.196 \mathrm{mmol}$, 1.0 equiv), $\mathrm{Et}_{3} \mathrm{~N}$ (0.204 mL, $1.46 \mathrm{mmol}, 7.4$ equiv) and $\mathrm{Ac}_{2} \mathrm{O}$ ( $0.092 \mathrm{~mL}, 0.973 \mathrm{mmol}, 4.0$ equiv). After stirring at ambient temperature for $2 \mathrm{~d}$ the reaction was terminated by addition of $\mathrm{H}_{2} \mathrm{O}$ and extracted with $\mathrm{Et}_{2} \mathrm{O}$ several times. The combined organic phases were dried over anhydrous $\mathrm{Na}_{2} \mathrm{SO}_{4}$, filtered, concentrated in vacuo. The residue was purified by silica gel column chromatography to give compound $\mathbf{1 6}$ (93 $\mathrm{mg}$, $0.17 \mathrm{mmol}, 88 \%) . R_{\mathrm{f}}=0.5$ (PE/EtOAc $3: 1$, visualized using an anisaldehyde or UV), $[\alpha]_{\mathrm{D}}{ }^{20}=+35.8\left(c 0.5, \mathrm{CH}_{2} \mathrm{Cl}_{2}\right) ;{ }^{1} \mathrm{H} \mathrm{NMR}$ $\left(400 \mathrm{MHz}, \mathrm{CDCl}_{3}, \mathrm{CHCl}_{3}=7.26 \mathrm{ppm}\right) \delta 7.41(\mathrm{~d}, J=8.6 \mathrm{~Hz}$, 2H), $7.27(\mathrm{~d}, J=9.0 \mathrm{~Hz}, 2 \mathrm{H}), 7.11(\mathrm{~d}, J=8.6 \mathrm{~Hz}, 2 \mathrm{H}), 6.89$ (d, $J=8.7 \mathrm{~Hz}, 2 \mathrm{H}), 6.01(\mathrm{~d}, J=3.2 \mathrm{~Hz}, 1 \mathrm{H}), 5.54(\mathrm{~s}, 1 \mathrm{H}), 4.44(\mathrm{~d}$, $J=10.8 \mathrm{~Hz}, 1 \mathrm{H}), 4.37$ (d, $J=10.8 \mathrm{~Hz}, 1 \mathrm{H}), 3.82(\mathrm{~s}, 3 \mathrm{H}), 2.84$ (dd, $J=1.8,12.3 \mathrm{~Hz}, 1 \mathrm{H}), 2.65(\mathrm{br}, 1 \mathrm{H}), 2.32(\mathrm{~s}, 3 \mathrm{H}), 2.24$ (d, $J$ $=2.6 \mathrm{~Hz}, 1 \mathrm{H}), 2.14(\mathrm{~s}, 3 \mathrm{H}), 2.10-2.06(\mathrm{~m}, 1 \mathrm{H}), 2.03-2.00(\mathrm{~m}$, $2 \mathrm{H}), 1.73(\mathrm{~s}, 3 \mathrm{H}), 1.60(\mathrm{~d}, J=14.0 \mathrm{~Hz}, 1 \mathrm{H}), 1.28(\mathrm{~s}, 3 \mathrm{H})$, $1.24(\mathrm{~s}, 3 \mathrm{H}), 1.11(\mathrm{~s}, 3 \mathrm{H}) ;{ }^{13} \mathrm{C} \mathrm{NMR}(100 \mathrm{MHz}$, $\left.\mathrm{CDCl}_{3}=77.16 \mathrm{ppm}\right) \delta 170.3,169.3,158.9,149.9,137.0,134.5$, 131.7, 128.7, 126.9, 122.7, 121.5, 113.8, 85.4, 81.0, 74.9, 71.0, $64.9,55.7,55.3,53.5,46.1,45.8,37.9,34.6,29.9,23.8,22.8$, 21.6, 21.2, 21.1; HRMS (ESI) $\mathrm{m} / z:[\mathrm{M}+\mathrm{Na}]^{+}$calculated for $\mathrm{C}_{34} \mathrm{H}_{40} \mathrm{O}_{6} \mathrm{Na}, 567.2723$; found, 567.2728.

\section{Synthesis of vinyl iodide 17}

To a stirred solution of compound of $\mathbf{1 6}(88 \mathrm{mg}, 0.162 \mathrm{mmol}$, 1.0 equiv) in acetone $(4 \mathrm{~mL})$ was added $\mathrm{AgNO}_{3}(8.3 \mathrm{mg}$, $0.05 \mathrm{mmol}, 0.3$ equiv) and $N$-iodosuccinimide $(40.0 \mathrm{mg}$, $0.18 \mathrm{mmol}, 1.1$ equiv) at ambient temperature. The resulting mixture was stirred for $1.5 \mathrm{~h}$ and concentrated in vacuo. The residue was purified by silica gel column chromatography to give the corresponding alkynyl iodide $(91.0 \mathrm{mg}, 0.136 \mathrm{mmol}$, $84 \%$ ). This product ( $81.9 \mathrm{mg}, 0.12 \mathrm{mmol}, 1.0$ equiv) was dissolved in a mixture of THF $(1.1 \mathrm{~mL})$ and $\mathrm{PrOH}(1.1 \mathrm{~mL}) . \mathrm{Et}_{3} \mathrm{~N}$ ( $0.026 \mathrm{~mL}, 0.183 \mathrm{mmol}, 1.5$ equiv) and 2-nitrobenzenesulfonyl hydrazide (34.5 mg, $0.159 \mathrm{mmol}, 1.3$ equiv) were sequentially added. After stirring for $20 \mathrm{~h}, \mathrm{Et}_{3} \mathrm{~N}(0.026 \mathrm{~mL}, 0.183 \mathrm{mmol}$, 1.5 equiv) and 2-nitrobenzenesulfonyl hydrazide $(17.25 \mathrm{mg}$, $0.079 \mathrm{mmol}, 0.7$ equiv) were added and the reaction mixture was stirred for additional $5 \mathrm{~h}$. The volatiles were then removed in vacuo at ambient temperature. The residue was purified by silica gel column chromatography to give vinyl iodide $\mathbf{1 7}$
(62.0 mg, $0.092 \mathrm{mmol}, 77 \%) . R_{\mathrm{f}}=0.60$ (PE/EtOAc 3:1, visualized using an anisaldehyde stain or UV), $[\alpha]_{\mathrm{D}}{ }^{20}=+14.1$ (c 0.95 , $\mathrm{CH}_{2} \mathrm{Cl}_{2}$ ); ${ }^{1} \mathrm{H} \mathrm{NMR}\left(400 \mathrm{MHz}, \mathrm{CDCl}_{3}, \mathrm{CHCl}_{3}=7.26 \mathrm{ppm}\right) \delta$ $7.28(\mathrm{~d}, J=8.6 \mathrm{~Hz}, 2 \mathrm{H}), 7.17(\mathrm{~d}, J=8.6 \mathrm{~Hz}, 2 \mathrm{H}), 7.11(\mathrm{~d}, J=$ $8.6 \mathrm{~Hz}, 2 \mathrm{H}), 6.90(\mathrm{~d}, J=8.6 \mathrm{~Hz}, 2 \mathrm{H}), 6.43(\mathrm{dd}, J=7.8,9.4 \mathrm{~Hz}$, $1 \mathrm{H}), 6.29(\mathrm{~d}, J=7.5 \mathrm{~Hz}, 1 \mathrm{H}), 6.03(\mathrm{~d}, J=2.5 \mathrm{~Hz}, 1 \mathrm{H}), 5.56(\mathrm{~s}$, $1 \mathrm{H}), 4.45(\mathrm{~d}, J=10.8 \mathrm{~Hz}, 1 \mathrm{H}), 4.38(\mathrm{~d}, J=10.8 \mathrm{~Hz}, 1 \mathrm{H}), 3.82$ (s, 3H), 2.89-2.83 (m, 2H), $2.37(\mathrm{ddd}, J=2.9,3.7,10.9 \mathrm{~Hz}$, $1 \mathrm{H}), 2.31(\mathrm{~s}, 3 \mathrm{H}), 2.15(\mathrm{~s}, 3 \mathrm{H}), 2.07(\mathrm{~d}, J=14.0 \mathrm{~Hz}, 1 \mathrm{H}), 1.79$ (t, $J=11.8 \mathrm{~Hz}, 1 \mathrm{H}), 1.64(\mathrm{~s}, 3 \mathrm{H}), 1.59$ (d, $J=14.0 \mathrm{~Hz}, 1 \mathrm{H})$, $1.30(\mathrm{~s}, 3 \mathrm{H}), 1.24$ (s, 3H), 1.09 (s, 3H); ${ }^{13} \mathrm{C} \mathrm{NMR}(100 \mathrm{MHz}$, $\left.\mathrm{CDCl}_{3}=77.16 \mathrm{ppm}\right) \delta 169.9,169.3,158.9,149.6,141.9,136.1$, 135.2, 131.7, 128.7, 126.7, 123.7, 121.9, 113.8, 80.9, 80.1, 75.5, 64.9, 55.9, 55.3, 53.4, 46.6, 46.1, 46.0, 38.3, 30.1, 23.5, 22.7, 22.2, 21.3, 21.2; HRMS (ESI) $\mathrm{m} / \mathrm{z}:[\mathrm{M}+\mathrm{Na}]^{+}$calculated for $\mathrm{C}_{34} \mathrm{H}_{41} \mathrm{O}_{6} \mathrm{INa}, 695.1846$; found, 695.1837 .

\section{Synthesis of vinyl iodide $\mathbf{1 8}$}

DDQ (56.5 mg, $0.25 \mathrm{mmol}, 3.0$ equiv) was added to a stirred solution of 17 (55.8 mg, $0.083 \mathrm{mmol}, 1.0$ equiv) in $\mathrm{CH}_{2} \mathrm{Cl}_{2}$ $(4.5 \mathrm{~mL}) / \mathrm{pH} 7.0$ phosphate buffer $(0.45 \mathrm{~mL})$ at $0{ }^{\circ} \mathrm{C}$. After stirring for $1.5 \mathrm{~h}$, the reaction mixture was terminated by addition of a saturated, aqueous $\mathrm{NaHCO}_{3}$ solution. The aqueous solution was extracted with $\mathrm{Et}_{2} \mathrm{O}$. The combined, organic phases were dried over anhydrous $\mathrm{Na}_{2} \mathrm{SO}_{4}$, filtered, concentrated in vacuo and the crude product was purified by silica gel column chromatography to furnish the title vinyl iodide $\mathbf{1 8}(40.0 \mathrm{mg}$, $0.072 \mathrm{~mol}, 87 \%) . R_{\mathrm{f}}=0.25$ (PE/EtOAc 3:1, visualized using an anisaldehyde or UV), $[\alpha]_{\mathrm{D}}{ }^{20}=+36.7\left(c \quad 0.86, \mathrm{CH}_{2} \mathrm{Cl}_{2}\right)$; ${ }^{1} \mathrm{H}$ NMR (400 MHz, $\left.\mathrm{CDCl}_{3}, \mathrm{CHCl}_{3}=7.26 \mathrm{ppm}\right) \delta 7.17$ (d, $J=$ $8.6 \mathrm{~Hz}, 2 \mathrm{H}), 7.11(\mathrm{~d}, J=8.6 \mathrm{~Hz}, 2 \mathrm{H}), 6.40$ (dd, $J=7.6,9.6 \mathrm{~Hz}$, $1 \mathrm{H}), 6.28(\mathrm{~d}, J=7.6 \mathrm{~Hz}, 1 \mathrm{H}), 6.03(\mathrm{~d}, J=2.3 \mathrm{~Hz}, 1 \mathrm{H}), 5.51(\mathrm{~s}$, $1 \mathrm{H}), 2.84(\mathrm{dd}, J=4.1,9.6 \mathrm{~Hz}, 1 \mathrm{H}), 2.70(\mathrm{~d}, J=12.7 \mathrm{~Hz}, 1 \mathrm{H})$, $2.34(\mathrm{ddd}, J=2.8,4.0,11.3 \mathrm{~Hz}, 1 \mathrm{H}), 2.31$ (s, 3H), 2.15 (s, 3H), $1.84(\mathrm{~d}, J=14.2 \mathrm{~Hz}, 1 \mathrm{H}), 1.77$ (d, $J=14.2 \mathrm{~Hz}, 1 \mathrm{H}), 1.74$ (t, $J$ $=11.9 \mathrm{~Hz}, 1 \mathrm{H}), 1.65(\mathrm{~s}, 3 \mathrm{H}), 1.30(\mathrm{~s}, 3 \mathrm{H}), 1.19(\mathrm{~s}, 3 \mathrm{H}), 1.07(\mathrm{~s}$, $3 \mathrm{H}) ;{ }^{13} \mathrm{C} \mathrm{NMR}\left(100 \mathrm{MHz}, \mathrm{CDCl}_{3}=77.16 \mathrm{ppm}\right) \delta 169.9,169.3$, 149.6, 141.8, 136.1, 135.7, 126.7, 122.4, 121.9, 80.1, 76.2, 75.4, 60.1, 54.8, 46.4, 46.3, 45.9, 38.1, 30.2, 26.4, 23.9, 22.2, 21.2, 21.1; HRMS (ESI) $m / z:[\mathrm{M}+\mathrm{Na}]^{+}$calculated for $\mathrm{C}_{26} \mathrm{H}_{33} \mathrm{O}_{5} \mathrm{INa}$, 575.1270; found, 575.1272 .

\section{Synthesis of triene 19}

To a stirred solution of vinyl iodide $\mathbf{1 8}(21 \mathrm{mg}, 40.2 \mu \mathrm{mol}$, 1.0 equiv) and boronate 13 (39.12 mg, $60.0 \mu \mathrm{mol}, 1.5$ equiv) in THF ( $5 \mathrm{~mL})$ and $\mathrm{H}_{2} \mathrm{O}(1.25 \mathrm{~mL})$ were sequentially added thallium(I) carbonate $(33.92 \mathrm{mg}, 72.0 \mu \mathrm{mol}, 1.8$ equiv) and $\mathrm{Pd}\left(\mathrm{PPh}_{3}\right)_{4}(13.93 \mathrm{mg}, 12.0 \mu \mathrm{mol}, 0.3$ equiv) at room temperature. The reaction mixture was stirred for $4 \mathrm{~h}$ and then $\mathrm{H}_{2} \mathrm{O}$ was added. The aqueous solution was extracted with $\mathrm{Et}_{2} \mathrm{O}$. The combined, organic phases were dried over anhydrous $\mathrm{Na}_{2} \mathrm{SO}_{4}$, 
filtered, concentrated in vacuo and the crude product was purified by silica gel column chromatography to furnish the title triene $19(29.3 \mathrm{mg}, 31.0 \mu \mathrm{mol}, 77 \%) . R_{\mathrm{f}}=0.4(\mathrm{PE} /$ EtOAc $2: 1$, visualized using an anisaldehyde or UV), $[\alpha]_{\mathrm{D}}{ }^{20}=+77.2$ (c $\left.0.53, \mathrm{CH}_{2} \mathrm{Cl}_{2}\right) ;{ }^{1} \mathrm{H}$ NMR $\left(400 \mathrm{MHz}\right.$, acetone- $d_{6}$, acetone- $\left.d_{5}=2.05 \mathrm{ppm}\right) \delta 7.32(\mathrm{~d}, J=15.7 \mathrm{~Hz}, 1 \mathrm{H}, \mathrm{H} 3), 7.19$ (d, $J=8.5 \mathrm{~Hz}, 2 \mathrm{H}, \mathrm{H} 27), 7.00$ (d, $J=8.5 \mathrm{~Hz}, 2 \mathrm{H}, \mathrm{H} 28), 6.64$ (dd, $J=11.7,14.1 \mathrm{~Hz}, 1 \mathrm{H}, \mathrm{H} 12), 6.17$ (dd, $J=10.9,11.2 \mathrm{~Hz}$, 1H, H14), 6.11-6.04 (m, 3H, H5, H11, H13), 6.01 (d, $J=2.6$ $\mathrm{Hz}, 1 \mathrm{H}, \mathrm{H} 25), 5.83$ (d, $J=15.7 \mathrm{~Hz}, 1 \mathrm{H}, \mathrm{H} 2), 5.78$ (d, $J=10.4$, $10.7 \mathrm{~Hz}, 1 \mathrm{H}, \mathrm{H} 15), 5.55$ (s,1H, H18), 5.48 (t, $J=10.1 \mathrm{~Hz}, 1 \mathrm{H}$, H10), 4.71 (dd, $J=6.6,9.2 \mathrm{~Hz}, 1 \mathrm{H}, \mathrm{H} 9), 4.14$ (q, $J=7.1 \mathrm{~Hz}$, 2H, H1'), 4.05-4.00 (m, 1H, H7), 2.87-2.88 (m, 1H, H16), 2.74 (d, $J=12.6 \mathrm{~Hz}, 1 \mathrm{H}, \mathrm{H} 19), 2.49$ (t, $J=6.9 \mathrm{~Hz}, 2 \mathrm{H}, \mathrm{H} 6), 2.39$ (ddd, $J=3.3,3.5,11.4 \mathrm{~Hz}, 1 \mathrm{H}, \mathrm{H} 24), 2.27$ (s, 3H, H12'), 2.12 (s, 3H, H10'), 1.93 (dd, $J=11.8,11.9 \mathrm{~Hz}, 1 \mathrm{H}, \mathrm{H} 23$ ), 1.89 (s, $3 \mathrm{H}, \mathrm{H} 3$ '), 1.84 (d, $J=13.6 \mathrm{~Hz}, 1 \mathrm{H}, \mathrm{H} 21 \mathrm{~b}), 1.83-1.80$ (m, 1H, H8), 1.73 (d, $J=13.9 \mathrm{~Hz}, 1 \mathrm{H}, \mathrm{H} 21 \mathrm{a}), 1.45$ (s, 3H, H5'), 1.30 (s, 3H, H6'), 1.23 (t, $J=7.1 \mathrm{~Hz}, 3 \mathrm{H}, \mathrm{H} 2$ ') 1.14 (s, 3H, H8'), 1.08 (d, $\left.J=6.8 \mathrm{~Hz}, 3 \mathrm{H}, \mathrm{H} 4^{\prime}\right), 1.07$ (s, 3H, H7'), 1.04-0.99 (m, 18H, H14', H16'), 0.70-0.58 (m, 12H, H13', H15'); ${ }^{13} \mathrm{C}$ NMR (400 MHz, acetone- $d_{6}=29.8$ and $\left.206.3 \mathrm{ppm}\right) \delta 169.4$ (C9'), 168.7 (C11'), 166.4 (C1), 149.8 (C29), 148.8 (C3), 139.3 (C5), 137.1 (C26), 134.9 (C17), 134.5 (C15), 133.8 (C4), 133.2 (C10), 131.6 (C13), 129.4 (C11), 127.1 (C12), 126.5 (C27), 125.6 (C14), 122.7 (C18),121.3 (C28), 115.9 (C2), 75.0 (C25), 74.8 (C20), 72.9 (C7), 70.1 (C9), 60.2 (C21), 59.6 (C1'), 55.1 (C19), 47.4 (C8), 46.6 (C24), 44.7 (C23), 40.8 (C16), 37.5 (C22), 32.6 (C6), 29.8 (C7'), 25.7 (C8'), 23.6 (C6'), 20.6 (C5'), 20.3 (C12'), 20.1 (C10'), 13.7 (C2'), 11.9 (C3'), 9.5 (C4'), 6.5 (C14'), 6.3 (C16'), 4.9 (C13',15'); HRMS (ESI) m/z: [M+ Na] ${ }^{+}$ calculated for $\mathrm{C}_{55} \mathrm{H}_{86} \mathrm{O}_{9} \mathrm{Si}_{2} \mathrm{Na}$, 969.5708; found, 969.5707 .

\section{Synthesis of elansolid B1 (2)}

Polyene 19 (2.65 mg, $2.79 \mu \mathrm{mol}, 1.0$ equiv) was dissolved in THF $(0.5 \mathrm{~mL})$ and cooled to $0{ }^{\circ} \mathrm{C}$. A solution of hydrogen fluoride pyridine complex $(0.5 \mathrm{~mL})$ prepared by mixing hydrogen fluoride pyridine ( $2 \mathrm{~mL}$; hydrogen fluoride $\approx 70 \%$ ) with pyridine $(5.6 \mathrm{~mL})$ in THF $(9.8 \mathrm{~mL})$ at $0{ }^{\circ} \mathrm{C}$. The reaction mixture was stirred for $1 \mathrm{~h}$ at this temperature and the reaction was terminated by addition of a saturated bicarbonate solution. The aqueous solution was extracted with $\mathrm{Et}_{2} \mathrm{O}$ for three times. The combined organic phases were dried over $\mathrm{Na}_{2} \mathrm{SO}_{4}$, filtered and concentrated under reduced pressure to afford the corresponding diol suitably pure for directly being employed in the next step. An aqueous solution of $\mathrm{LiOH}(1 \mathrm{M}, 0.3 \mathrm{~mL}, 107$ equiv) was added to crude diol in iPrOH $(0.3 \mathrm{~mL})$ and THF $(0.3 \mathrm{~mL})$ at room temperature. After stirring for $5 \mathrm{~h}$, the reaction was terminated by slowly adding $\mathrm{HCl}(1 \mathrm{~N}, 0.24 \mathrm{~mL})$, phosphate buffer $(\mathrm{pH} 7,0.1 \mathrm{~mL})$ and $\mathrm{MeOH}(0.3 \mathrm{~mL})$. The crude mixture was directly subjected to HPLC (C18 ISIS-SP) (MeOH: $\mathrm{H}_{2} \mathrm{O} /$
$50 \mathrm{mM} \mathrm{NH}_{4} \mathrm{OAc} 70: 30$ to $\mathrm{MeOH}: \mathrm{H}_{2} \mathrm{O} / 50 \mathrm{mM} \mathrm{NH}_{4} \mathrm{OAc}$ 100:0 \{0-70 $\mathrm{min}\}, 3.0 \mathrm{~mL} / \mathrm{min})$ to give elansolid B1 (2) $(0.88 \mathrm{mg}$, $1.45 \mu \mathrm{mol}, 52 \%$ over two steps, $\left.t_{\mathrm{R}}=47.8 \mathrm{~min}\right) .[\alpha]_{\mathrm{D}}{ }^{20}=+176.0$ (c $0.05, \mathrm{MeOH}) ;{ }^{1} \mathrm{H}$ NMR $\left(500 \mathrm{MHz}\right.$, acetone- $d_{6}$; acetone- $\left.d_{5}=2.05 \mathrm{ppm}\right) \delta 7.35(\mathrm{~d}, J=15.6 \mathrm{~Hz}, 1 \mathrm{H}, \mathrm{H} 3), 7.14$ (d, $J=8.3 \mathrm{~Hz}, 2 \mathrm{H}, \mathrm{H} 27), 6.75$ (d, $J=8.5 \mathrm{~Hz}, 2 \mathrm{H}, \mathrm{H} 28), 6.57$ (dd, $J=12.2,13.6 \mathrm{~Hz}, 1 \mathrm{H}, \mathrm{H} 12), 6.17$ (t, $J=7.23 \mathrm{~Hz}, 1 \mathrm{H}, \mathrm{H} 5)$, 6.09-6.02 (m, 2H, H13, H14), 5.99 (dd, $J=10.8,11.0 \mathrm{~Hz}, 1 \mathrm{H}$, $\mathrm{H} 11), 5.84(\mathrm{~d}, J=15.7 \mathrm{~Hz}, 1 \mathrm{H}, \mathrm{H} 2), 5.70(t, J=10.5 \mathrm{~Hz}, 1 \mathrm{H}$, H15), 5.55 (dd, $J=9.5,10.9 \mathrm{~Hz}, 1 \mathrm{H}, \mathrm{H} 10), 5.52$ (s, 1H, H18), $5.18(\mathrm{~d}, J=2.5 \mathrm{~Hz}, 1 \mathrm{H}, \mathrm{H} 25), 4.94(\mathrm{ddd}, J=0.9,3.6,8.6 \mathrm{~Hz}$, 1H, H9), 3.87-3.84 (m, 1H, H7), 2.92 (dd, $J=4.0,10.8 \mathrm{~Hz}, 1 \mathrm{H}$, H16), 2.69 (d, $J=2.6 \mathrm{~Hz}, 1 \mathrm{H}, \mathrm{H} 19), 2.59$ (ddd, $J=4.2,6.8$, $15.3 \mathrm{~Hz}, 1 \mathrm{H}, \mathrm{H6a}), 2.51-2.47$ (m, 1H, H6b), 2.22-2.18 (m, 1H, H24), 1.99-1.95 (m, 1H, H23), 1.88 (s, 3H, H1'), 1.83-1.80 (m, $1 \mathrm{H}, \mathrm{H} 8), 1.83$ (d, $J=13.8 \mathrm{~Hz}, 1 \mathrm{H}, \mathrm{H} 21 \mathrm{a}), 1.73$ (d, $J=13.8 \mathrm{~Hz}$, 1H, H21b), 1.44 (dd, $J=1.4,2.2 \mathrm{~Hz}, 3 \mathrm{H}, \mathrm{H} 3$ '), 1.30 (s, 3H, H5'), 1.29 (s, 3H, H6'), 1.14 (s, 3H, H4'), 1.03 (d, $J=7.0 \mathrm{~Hz}$, $3 \mathrm{H}, \mathrm{H} 2$ ') ${ }^{13} \mathrm{C}$ NMR (125 MHz, acetone- $d_{6}=29.8$ and 206.3 ppm) $\delta 167.5$ (C1), 155.7 (C29), 149.4 (C3), 138.9 (C5), 135.2 (C26, C17), 134.8 (C15), 133.8 (C4), 132.9 (C10), 130.9 (C13), 129.3 (C11), 127.5 (C12), 127.0 (C27), 126.5 (C14), 122.5 (C18), 115.9 (C2), 114.5 (C28), 74.9 (C20), 73.0 (C7), 72.2 (C25), 68.5 (C9), 60.5 (C21), 55.1 (C19), 48.4 (C24), 44.5 (C23), 44.0 (C8), 40.4 (C16), 37.7 (C22), 34.1 (C6), 30.9 (C5'), 25.7 (C4'), 23.8 (C6'), 20.6 (C3'), 11.8 (C1'), 10.7 (C2'); HRMS (ESI) $\mathrm{m} / \mathrm{z}:[\mathrm{M}+\mathrm{Na}]^{+}$calculated for $\mathrm{C}_{37} \mathrm{H}_{50} \mathrm{O}_{7} \mathrm{Na}$, 629.3454; found, 629.3463 .

\section{Synthesis of elansolid B2 (3)}

In aqueous solution of $\mathrm{LiOH}(1 \mathrm{M}, 0.3 \mathrm{~mL}, 107$ equiv) was added to the crude diol $(3.1 \mu \mathrm{mol})$ described for the synthesis of elansolid B1 (2) in MeOH (0.3 mL) and THF $(0.3 \mathrm{~mL})$ at room temperature. After stirring for $5 \mathrm{~h}$, the reaction was terminated by slowly adding $\mathrm{HCl}(1 \mathrm{~N}, 0.24 \mathrm{~mL})$, phosphate buffer ( $\mathrm{pH} 7$, $0.1 \mathrm{~mL})$ and $\mathrm{MeOH}(0.3 \mathrm{~mL})$. The crude mixture was directly subjected to HPLC (C18 ISIS-SP) ( $\mathrm{MeOH}: \mathrm{H}_{2} \mathrm{O} / 50 \mathrm{mM}$ $\mathrm{NH}_{4} \mathrm{OAc} 70: 30$ to $\mathrm{MeOH}: \mathrm{H}_{2} \mathrm{O} / 50 \mathrm{mM} \mathrm{NH}_{4} \mathrm{OAc}$ 100:0 \{0-70 $\mathrm{min}\}, 3.0 \mathrm{~mL} / \mathrm{min})$ to give elansolid $\mathrm{B} 2(3)(1.2 \mathrm{mg}$, $1.93 \mu \mathrm{mol}, 63 \%$ over two steps, $\left.t_{\mathrm{R}}=63.4 \mathrm{~min}\right) .[\alpha]_{\mathrm{D}}{ }^{20}=+262.1$ (c $0.087, \mathrm{MeOH}) ;{ }^{1} \mathrm{H}$ NMR $\left(400 \mathrm{MHz}\right.$, acetone- $d_{6}$; acetone- $\left.d_{5}=2.05 \mathrm{ppm}\right) \delta 7.35(\mathrm{~d}, J=15.7 \mathrm{~Hz}, 1 \mathrm{H}, \mathrm{H} 3), 7.07$ (d, $J=8.4 \mathrm{~Hz}, 2 \mathrm{H}, \mathrm{H} 27), 6.77$ (d, $J=8.5 \mathrm{~Hz}, 2 \mathrm{H}, \mathrm{H} 28), 6.63$ (dd, $J=11.5,14.6 \mathrm{~Hz}, 1 \mathrm{H}, \mathrm{H} 12), 6.34$ (dd, $J=11.5,14.6 \mathrm{~Hz}$, 1H, H13), 6.18 (t, $J=7.2 \mathrm{~Hz}, 1 \mathrm{H}, \mathrm{H} 5), 6.13$ (t, $J=11.2 \mathrm{~Hz}$, 1H, H11), 6.02 (t, $J=11.2 \mathrm{~Hz}, 1 \mathrm{H}, \mathrm{H} 14), 5.82$ (d, $J=15.7 \mathrm{~Hz}$, $1 \mathrm{H}, \mathrm{H} 2), 5.68$ (t, $J=10.8 \mathrm{~Hz}, 1 \mathrm{H}, \mathrm{H} 15), 5.57$ (dd, $J=9.7,10.1$ $\mathrm{Hz}, 1 \mathrm{H}, \mathrm{H} 10$ ), 5.48 (s, 1H, H18), 4.99 (ddd, $J=0.9,3.5,8.6$ $\mathrm{Hz}, 1 \mathrm{H}, \mathrm{H} 9), 4.66$ (d, $J=2.4 \mathrm{~Hz}, 1 \mathrm{H}, \mathrm{H} 25), 3.88-3.84$ (m, 1H, H7), 3.12 (s, 3H, H7'), 2.90 (dd, $J=3.3,10.6 \mathrm{~Hz}, 1 \mathrm{H}, \mathrm{H} 16)$, 2.64-2.62 (m, 1H, 19H), 2.60-2.58 (m, 1H, H6a), 2.52-2.44 
(m, 1H, H6b), 2.01-1.97 (m, 1H, H24), 1.95-1.91 (m, 1H, H23), 1.89 (s, 3H, H1'), 1.85-1.82 (m, 1H, H8), 1.82 (d, $J=$ $13.5 \mathrm{~Hz}, 1 \mathrm{H}, \mathrm{H} 21 \mathrm{a}$ ), 1.72 (d, $J=13.8 \mathrm{~Hz}, 1 \mathrm{H}, \mathrm{H} 21 \mathrm{~b}$ ), 1.45 (dd, $J=1.3,2.1 \mathrm{~Hz}, 3 \mathrm{H}, \mathrm{H} 3$ '), 1.29 (s, 3H, H5'), 1.21 (s, 3H, H6'), 1.12 (s, 3H, H4'), 1.03 (d, $J=7.0 \mathrm{~Hz}, 3 \mathrm{H}, \mathrm{H} 2$ '); ${ }^{13} \mathrm{C} \mathrm{NMR}$ $\left(100 \mathrm{MHz}\right.$, acetone- $d_{6}=29.8$ and $\left.206.3 \mathrm{ppm}\right) \delta 167.3(\mathrm{C} 1)$, 156.2 (C29), 149.4 (C3), 139.0 (C5), 135.5 (C15), 135.3 (C17), 133.8 (C4), 132.5 (C10), 131.6 (C13), 131.0 (C26), 129.4 (C11), 128.3 (C27), 126.8 (C12), 124.3 (C14), 122.5 (C18), 115.7 (C2), 114.7 (C28), 82.9 (C25), 74.8 (C20), 73.0 (C7), 68.5 (C9), 60.4 (C21), 55.6 (C7'), 55.2 (C19), 48.8 (C24), 44.8 (C23), 44.0 (C8), 40.3 (C16), 37.6 (C22), 34.2 (C6), 30.4 (C6'), 25.7 (C4'), 23.7 (C5'), 20.9 (C3'), 11.8 (C1'), 10.8 (C2'); HRMS (ESI) $m / z$ : $[\mathrm{M}+\mathrm{Na}]^{+}$calculated for $\mathrm{C}_{38} \mathrm{H}_{52} \mathrm{O}_{7} \mathrm{Na}$, 643.3611; found, 643.3611 .

\section{Supporting Information}

\section{Supporting Information File 1}

${ }^{1} \mathrm{H}$ and ${ }^{13} \mathrm{C}$ NMR spectra of synthesized compounds. [http://www.beilstein-journals.org/bjoc/content/ supplementary/1860-5397-13-124-S1.pdf]

\section{References}

1. Gerth, K.; Steinmetz, H.; Höfle, G. Elansolids, novel natural metabolites of flexibacter and antibiotically active derivatives thereof. Eur. Pat. Appl. EP 2093212 A1, Aug 26, 2009.

2. Steinmetz, H.; Gerth, K.; Jansen, R.; Schläger, N.; Dehn, R.; Reinecke, S.; Kirschning, A.; Müller, R. Angew. Chem. 2011, 123, 553-557. doi:10.1002/ange.201005226

3. Dehn, R.; Katsuyama, Y.; Weber, A.; Gerth, K.; Jansen, R.; Steinmetz, H.; Höfle, G.; Müller, R.; Kirschning, A. Angew. Chem., Int. Ed. 2011, 123, 3968-3973. doi:10.1002/ange.201006880

4. Jansen, R.; Gerth, K.; Steinmetz, H.; Reinecke, S.; Kessler, W.; Kirschning, A.; Müller, R. Chem. - Eur. J. 2011, 17, 7739-7744. doi:10.1002/chem.201100457

5. Steinmetz, H.; Zander, W.; Shushni, M. A. M.; Jansen, R.; Gerth, K.; Dehn, R.; Dräger, G.; Kirschning, A.; Müller, R. ChemBioChem 2012, 13, 1813-1817. doi:10.1002/cbic.201200228

6. Minami, A.; Oikawa, H. J. Antibiot. 2016, 69, 500-506. doi:10.1038/ja.2016.67

7. Weber, A.; Dehn, R.; Schläger, N.; Dieter, B.; Kirschning, A. Org. Lett. 2014, 16, 568-571. doi:10.1021/ol403441c

8. For simplification the carbon atoms are numbered as in the natural product elansolid throughout the text.

9. Wang, L. L.; Candito, D.; Dräger, G.; Hermann, J.; Müller, R.; Kirschning, A. Chem. - Eur. J. 2017, 23, 5291-5298. doi:10.1002/chem.201605884

10. Jones, G. R.; Landais, Y. Tetrahedron 1996, 52, 7599-7662. doi:10.1016/S0040-4020(96)00038-5

11. Hartmann, O.; Kalesse, M. Org. Lett. 2012, 14, 3064-3067. doi:10.1021/ol3011387
12. Gao, D.; O'Doherty, G. A. Org. Lett. 2010, 12, 3752-3755. doi:10.1021/ol101340n

13. Fuwa, H.; Sasaki, M. Org. Lett. 2010, 12, 584-587. doi:10.1021/ol902778y

14. Fuwa, H.; Suzuki, T.; Kubo, H.; Yamori, T.; Sasaki, M. Chem. - Eur. J. 2011, 17, 2678-2688. doi:10.1002/chem.201003135

15. Essig, S.; Bretzke, S.; Müller, R.; Menche, D. J. Am. Chem. Soc. 2012, 134, 19362-19365. doi:10.1021/ja309685n

\section{License and Terms}

This is an Open Access article under the terms of the Creative Commons Attribution License

(http://creativecommons.org/licenses/by/4.0), which permits unrestricted use, distribution, and reproduction in any medium, provided the original work is properly cited.

The license is subject to the Beilstein Journal of Organic Chemistry terms and conditions:

(http://www.beilstein-journals.org/bjoc)

The definitive version of this article is the electronic one which can be found at: doi:10.3762/bjoc. 13.124 